\title{
A Novel Ultrasonic Water Pollution Detector Based on WSN Yili Wang ${ }^{1}$ a*$^{*}$, Jianguo Gong ${ }^{1}$, Shuwen Jin ${ }^{1}$, Yongjie Lu ${ }^{1}$, Chuanrui Mei ${ }^{1}$ and
Zhongwei Zhao
}

${ }^{1}$ IEE Dept. Zhejiang Gongshang University, 310018, Hangzhou , China

a1065717808@qq.com

Keywords: Ultrasonic detector; WSN; Curve squares filter; Vector-difference

\begin{abstract}
This paper proposes a novel ultrasonic detector of water pollution based on WSN. The proposed vector difference algorithm on one time period can precisely calculate the velocity and height of drainage water pollution. The captured data with the detector will be transmitted to the server by WSN. Compared the popular detectors in the market, the detector in this paper is not only used to water pollution but used to any industry field if which need low-cost application or low-power remote detection.
\end{abstract}

\section{Introduction}

Industrial wastewater, as the main pollution source of water, has the characteristics of large quantity, wide range, complex composition, high toxicity and easy purification. The pollution of water resources is extremely huge in China. Although many advanced techniques have been used extensively in the water-flow measurement field, they have not progressed significantly in the above-mentioned measurements aspects. This action directly affects the intensity and effectiveness of monitoring. Environmental protection will be more important.

Ultrasonic flow-meter commonly is used as a measurement method to detect the water pollution According to different transmission speed; there are different algorithms to computer the pollution level, such as Doppler algorithm, noise-time algorithm and so on. Meanwhile, transmission speed difference also includes direct time difference, phase difference and frequency difference etc. Comparing to these different algorithms, the time difference algorithm is more commonly used because of simple computing way, which achieves the purpose of measuring the flow characteristics by the use of real-time captured data when acoustic waves are in the fluid the propagation velocity changes as the fluid velocity changes. However, there are some problems with the current time difference algorithm: It cannot accurately draw the water flow velocity and height vector relation that is hard to achieve real-time monition, the means is much more cumbersome. In addition, the accuracy of measurement is not good. Therefore, the market needs a high precision measurement, easy installation, wide application of the flow-meter.

This paper proposes a system which utilizes the ultrasonic wave-length in different time intervals to detect the vector difference based on a new 16-bit MSP430G2 series microcontroller. The presented time-domain algorithm can get the real-time water flow and height by using adjusts time different vector function; at the same time, the processed data is transmitted to the server through the wireless sensor network node controller zigbee deployed on the sensor side. Environmental monitoring personnel only through any terminal (mobile phone, PC, processor, etc.) log on to the server to obtain the monitoring point of the flow of information. The whole process is very convenient and safe. The whole test system integrates wireless sensor network technology, embedded system, AD / DA technology, web server technology, differential fusion technology and wireless data transmission technology into one, which is safe, convenient, mobile, intelligent and accurate. In addition, the system can be improved for urban and rural water and farmland irrigation water measurement, in order to obtain greater market efficiency. 


\section{Algorithm}

The system uses multi-sensor and microcontroller to achieve the flow of detection, the realization of the program shown in Fig. 1:

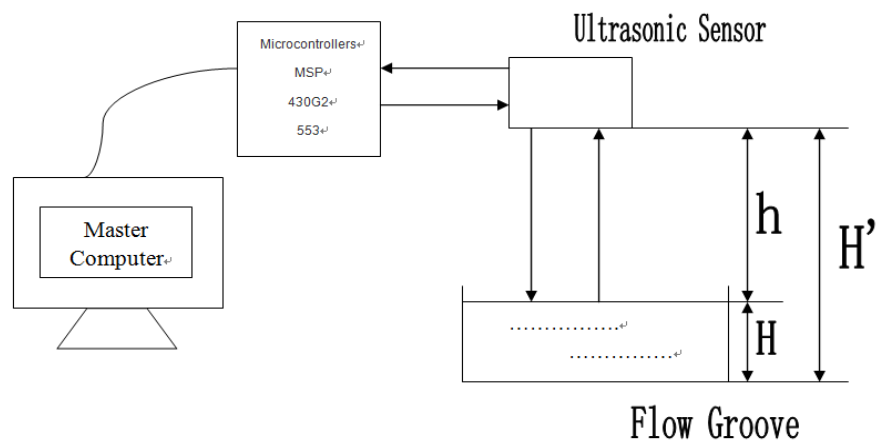

Figure 1. Principle of flow detection

The Flow detection algorithm based on time-domain difference is show as follow. We assume that when the water flow through the flow tank to form a natural flow, the relationship between the flow rate- $-\mathrm{Q}$, and the water level in the water tank- $-\mathrm{H}$. Here, the flow coefficient is related to the shape and size of the flow channel, and a corresponding value can be given according to the actual situation. As can be seen from Eq.1, as long as the (water level) measurement, you can indirectly get flow $\mathrm{Q}$. The measurement method of $\mathrm{H}$ as shown above, $\mathrm{H}$ will be expressed as follows:

$$
\mathrm{H}(\mathrm{t})=\mathrm{H}^{\prime}-\mathrm{ll}(\mathrm{t}) \text {. }
$$

Where $\mathrm{H}$ is the distance from sensor to zero water level of the flow tank, $h(t)$ is the distance from the sensor to the water level at the time. To get the $\mathrm{h}(\mathrm{t})$ need following algorithm procedure:

I: Excitation pulse issued by the microcontroller prompts the ultrasonic sensor to start sending ultrasound

II: The receiving end of the sensor is ready to receive the signal until it receives the reflected signal;

III: Using I2C interface to return the analog signal after the $\mathrm{A} / \mathrm{D}$ conversion to the microcontroller;

IV: The completion of the data collection is the data processing.

V: The time vector $t$ is required for the propagation of the ultrasonic wave is calculated.

The last above algorithm step will get the time vector $\mathrm{t}$ to fit following formula:

$$
\ln (\mathrm{t})=\frac{\mathrm{t} \times \mathrm{t}}{2}
$$

The speed of propagation of ultrasound in the air is $c$, with (2), $h(t)$ will be gotten. In order to further improve the accuracy of the data, the algorithm uses the software to accumulate the information to determine the continuous measurement of the data, in time, $\mathrm{T}$, to do a continuous average, so as to get in this period of time the level of the exact value of the level:

$$
\mathrm{H}=\frac{1}{\mathrm{~T}} \int_{0}^{\mathrm{T}} \mathrm{H}(\mathrm{t}) \mathrm{dt} .
$$

Taking into account the relationship between sound velocity and temperature, by using the temperature sensor deployed at the front end, enables the system to perform automatic temperature compensation to reduce unnecessary errors. The whole process is followed as Fig.2: 


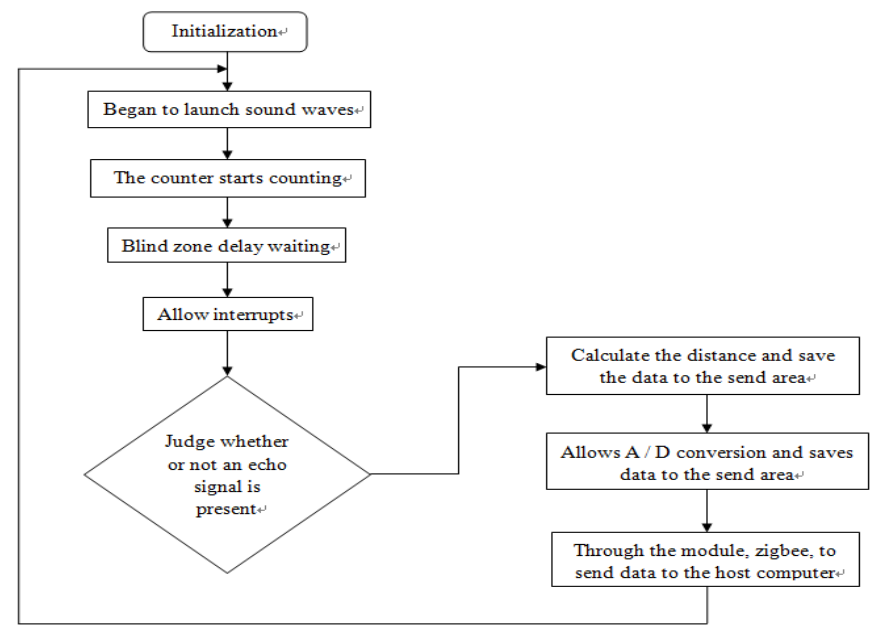

Figure 2. Algorithm procedure

\section{Detector Hard ware Implementation}

The hardware part mainly consists of front-end sensor (including ultrasonic sensor, temperature sensor and color sensor), power supply system, A / D conversion chip and micro-control unit, wireless transmission module zigbee and web server technology based back-end server. Among them, A / D conversion and microcontroller are the core part of the system. We use TI's ultra-low-power microcontroller MSP430G2553 as the core part of the system, which integrates two AD channels, and use its ultra-low power mode to complete the ultrasonic sensor and the A / D converted analog signal data processing tasks. As for the zigbee part, we use TI's CC2430, which has low power, speed, mode and more features. Here, A / D converter chip is the multi-sensor acquisition of analog signals into digital signals, and in order to achieve the desired results better, we require it to speed and can easily complete transmission with SCM data. Therefore, the system uses the ADE7816, a high-precision, multi-channel access through the SPI or I2C interface, on-chip metering register. At the same time, use its I2C interface to transmit data with the microcontroller. In this part, the power circuit uses National Semi's TD1583 dual DCDC to complete high-efficiency, low ripple power level conversion, and divide the input of the $+5 \mathrm{~V}$ power supply into two part, one for the system voltage of $3.3 \mathrm{~V}, 1.5 \mathrm{~A}$ current, and then through the inductor to complete analog power and digital power isolation, another for the GPRS, 4.0V voltage and 2.5A current, then GPRS module SIMCOM SIM900A module here, integrated tcp/ip protocol stack, through the 5-wire UART port and exchange data with microcontroller, and the rate is $115200 \mathrm{bps}$. In the process of the signal transmission, the system uses the wireless transmission module zigbee to achieve the transmission of signals to the master computer, and then uses the web server technology connects with the background server through the GPRS / WIFI / 3G wireless sensor network, and finally the water pollution flow information wireless transmission completes. It is worth mentioning that the system supplied by the solar panel power, reducing the possibility of secondary pollution and meanwhile reducing costs, but also eliminates the need for complex and cumbersome power supply unit, which makes it more convenient.

\section{Test Results}

Through the actual measurement, compare the data collected by the program with the data collected by the general time difference method (traditional algorithm) as the following table: 


\begin{tabular}{ccccc}
\hline time & $\begin{array}{c}\text { Actual data }(\text { height }(\mathrm{m}) \\
\text { and flow }\left(\mathrm{m}^{3} / \mathrm{s}\right)\end{array}$ & $\begin{array}{c}\text { data collected by the } \\
\text { program }\end{array}$ & $\begin{array}{c}\text { data collected by } \\
\text { the general time } \\
\text { difference method }\end{array}$ \\
\hline 1hour & $0.7 \quad ; \quad 12$ & $0.65 ; 11.82$ & $0.58 ; 11.32$ \\
8 hours & $0.5 \quad ; 10.5$ & $0.47 ; 10.33$ & $0.42 ; 9.87$ \\
24 hours & $0.6 \quad ; 10.8$ & $0.58 ; 10.45$ & $0.46 ; 10.39$ \\
48hours & $0.6 \quad ; 10.5$ & $0.57 ; 10.29$ & $0.52 ; 9.88$ \\
& $0.5 \quad ; 11$ & $0.47 ; 10.82$ & $0.39 ; 10.12$ \\
\hline
\end{tabular}

We can see from the measured data that the system's algorithm is closer to the actual data, which also shows that the data collected by the tester using the designed algorithm of will be more accurate.

\section{Conclusion}

A novel pollution detection system based on WSN is proposed in this paper. By using the curve-multiplication algorithm of the wavelength and time-dependent difference of the ultrasonic wave, the system can accurately calculate the flow and height of water pollution, Sensor node controller zigbee real-time transmission to the network data processing platform through the GPRS wireless network to send to the back-end server, so as to achieve real-time monitoring of water pollution monitoring point for the purpose of water pollution flow detection system. From the actual data collection point of view, the system measured data accuracy than the current market, the common water pollution detector to be much more accurate, and because the use of wireless sensor network, so the effect of remote monitoring, reducing the cost of manual participation And the error, has the very good market value and the scientific research value.

\section{References}

[1] D.Z. Tao, L. Wang, etc.: Acoustics and Electronic Engineering, (2001) NO.1, p.46 48.

[2] L. Zhai: Design of a Sewage Flow Measurement System (MS., Taiyuan University of Technology, China 2008).

[3] H.L. Zhu, etc.: Non - Contact Measurement of Pipeline Flow: Methods and Techniques (China Meteorological Press, China 1999), p.15 52.

[4] H.L. Zhu, X. Zhou: Non - Contact Measurement of Pipeline Flow: Methods and Techniques (China Meteorological Press, China 1999), p.15 16.

[5] X. Sheng: China Instrumentation, (2005) NO.7, p.98 99.

[6] X.B. Zhu, L.L. Xie, M.J. Tong: Journal of Transducer Technology, (2004) NO.1, p.65 66.

[7] C.C. Chen, Z.W. Zhao, etc.: Wireless Internet Technology, (2011) NO.12, p.37 38.

[8] X.H. Li: Environmental Engineering, (1996) NO.2, p.45 47.

[9] L.H. Li, K.M. Xie: Journal of Taiyuan University of Technology, (2001) NO.4, p.417 420.

[10] C.W. Li, P. Chen, M. Yuan: Metrology \& Measurement Science of Jiangsu Province (Jiangsu Province, China, 2008).

[11] Q.Q. Meng, D.W. Wu, Y. Lin, X. Zhong, H.T. Xian: China Water and Wastewater, (2004) NO.7, p.34 36.

[12]X.S. Wu, X.Y. Liao, L. Wang: Chinese Hydraulic Engineering Society (Guiyang Province, 
China, 2010).

[13] Y. Qi, Z.Z. Wang: Research on Automatic Monitoring System of Sewage Quality and Flow (MS., Taiyuan University of Technology, China 2009). 